\title{
Maximizing Network Lifetime by using ERMECR and ERMER Algorithms with Residual Energy
}

\author{
Agilan $S^{1}$, Gopinathan $B^{2}$ \\ PG scholar, Department of Computer Science and Engineering, Adhiyamaan College of Engineering, \\ Hosur, Krishnagiri (Dist), Tamilnadu, India ${ }^{1}$ \\ Associate Professor, Department of Computer Science and Engineering, Adhiyamaan College of Engineering, \\ Hosur, Krishnagiri (Dist), Tamilnadu, India ${ }^{2}$
}

\begin{abstract}
In wireless ad hoc network, the major problem is to provide reliable energy efficient routes which maximize the network lifetime, i.e., the number of successful transmissions. Residual energy metric is estimated for providing energy efficiency and improved reliability. In this paper, new energy-aware routing algorithms for wireless ad hoc networks, called Enhanced Reliable Minimum Energy Cost Routing (ERMECR) and Enhanced Reliable Minimum Energy Routing (ERMER) are proposed. ERMECR consider the remaining battery energy of nodes to find reliable routes and effectively reduce the energy consumption that increases the operational lifetime. In ERMECR, the energy consumed for retransmission of the packet as well, when the packet or its acknowledgment is lost. ERMER finds routes which minimizing the total energy required for end-to-end packet traversal. ERMECR and ERMER are to ensure reliability based on hop-by-hop and end-to-end retransmissions.
\end{abstract}

Keywords: Energy-aware routing, reliability, hop-by-hop and end-to-end retransmissions, wireless ad hoc networks

\section{INTRODUCTION}

Ad hoc network is a network formed without any central administration which consists of mobile nodes that use a wireless interface to send packet data. Wireless ad hoc network (WANET) is a decentralized wireless network and no preexisting infrastructure. Instead of each node in a network can serve as routers and hosts, they can forward packets for other nodes.

Energy consumption is also one of the most important performance metrics for wireless ad hoc networks, it directly relates to the operational lifetime of the networks. In the Wireless Ad-hoc Networks, battery replacement may not be possible. So as far as energy consumption concerned, should try to preserve energy while maintaining high connectivity. Each node depends on small low-capacity batteries as energy sources, and cannot expect replacement when operating in hostile and remote regions. For Wireless Ad hoc Networks, energy depletion and reduction is the primary factor in connectivity degradation and length of operational lifetime. Overall performance becomes highly dependent on the energy efficiency of the algorithm.

Effective mechanism for reducing the energy cost of forwarding the packet in wireless ad hoc network is done by energy efficient routing algorithms. In general, routes are discovered considering the energy consumed for end-to-end (E2E) packet traversal. This should not finding less reliable routes or overusing a set of specific nodes in the network. Energy-efficient routing in ad hoc networks is neither complete nor efficient without the consideration of reliability of links and remaining energy of nodes.
Finding reliable routes can enhance quality of the service. Whereas, considering the residual energy of nodes in routing can avoid nodes from being overused and can eventually lead to an increase in the operational lifetime of the network.

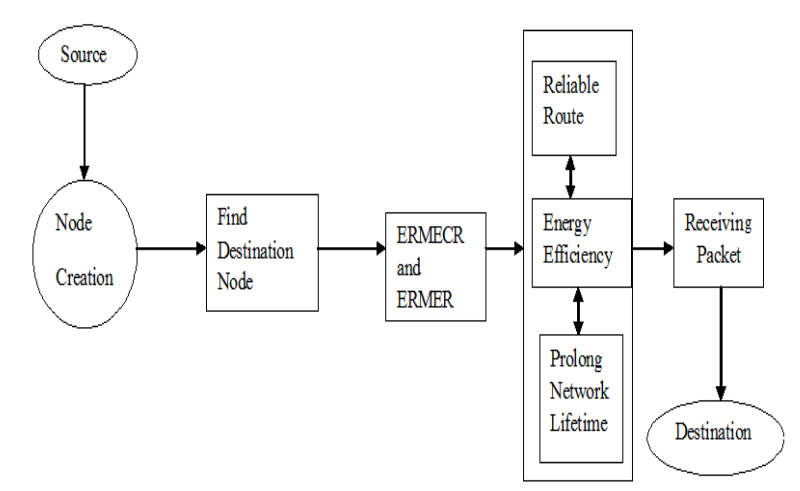

Fig. 1 Overall System Diagram

ERMECR and ERMER are able to find reliable routes, in which constituent links require less number of packet retransmissions due to packet loss. This in turn decreases the latency of packet delivery and saves energy as well. To prolong the network lifetime, power management and energy-efficient routing techniques become necessary.

The Fig. 1 represents the overview of our proposed system. The description of this diagram is given in section IV. 
II.

\section{RELATED WORK}

During the last decade, various routing algorithms have been proposed aiming at increasing energy-efficiency, reliability, and the lifetime of the networks. Broadly group them into three categories.

A. Reliability of Links

The first category of reliability of links is to find more reliable routes. For instance, De Couto et al. [1] introduced the notion of ETX (Expected Transmission Count) to find reliable routes that consist of links requiring less number of retransmissions for lost packet recovery. Although such routes may consume less energy since they require less number of retransmissions. They do not necessarily minimize the energy consumption for E2E packet traversal. A higher priority for reliability of routes may result in overusing some nodes and also some links more reliable than others, these links will repeatedly be used to forward packets.

\section{B. Energy-Efficient and Network Lifetime}

The second category includes algorithms that aim at finding energy-efficient routes [2], [3], [4], [5], [6], [7]. These algorithms do not consider the remaining battery energy of nodes to avoid overuse of nodes, even though some of them [4], [5], [6], [7] address energyefficiency and reliability together. Apart from this, many routing algorithms - including energy-efficient algorithms proposed [2], [3], [4], [5], [6], [7] in has a major drawback. It does not consider the actual energy consumption of nodes to discover energy efficient routes. It only considers the transmission power of nodes neglecting the energy consumed by processing elements of transmitters and receivers. This negatively affects energy efficiency, reliability, and the operational lifetime of the network altogether.

Energy-aware routing protocols for variable power scenarios aim to directly minimize the total power consumed over the entire transmission path. PAMAS (Power aware Multi access protocol with signaling) [2], is one such minimum total transmission energy protocol, where the link cost was set to the transmission power and Dijkstra's shortest path algorithm was used to compute the path that uses the smallest cumulative energy. In the case where nodes can dynamically adjust their power based on the link distance, such as formulation often leads to the formation of a path with a large number of hops.

Power-Aware Route Optimization (PARO) algorithm [3], [4] is designed for scenarios where the nodes can dynamically adjust their transmission power. PARO attempts to generate a path with a larger number of short-distance hops. According to the PARO protocol, a candidate intermediary node monitors an ongoing direct communication between two nodes and evaluates the potential for power savings by inserting itself in the forwarding path-infect, replacing the direct hop between the two nodes by two smaller hops through itself.

MMBCR [5] is a power-aware routing algorithm, which selects the path whose critical node has the highest residual battery energy. The node having the battery level, greater than the threshold value is considered as the strongest node. It selects the route with strongest node for packet transmission. Since the MMBCR algorithm never tries to minimize the total transmission energy along a path, it can lead overall higher energy consumption and consequently, a reduction of the average node lifetime.

CMMBCR[6] algorithm uses the minimum energy path initially, as long as the battery power level on all the nodes in the selected path lies above a certain threshold. Once one or more of nodes on all possible paths falls below this battery protection threshold, the algorithm switches to the MMBCR mode. This results in overall higher energy consumption but provides higher reliability.

ERS [9] is applied to AODV for route discovery process to reduce overhead and to use energy efficiently by using the Time to Live (TTL) mechanism. The ERS belongs to the reactive protocols. The goal of ERS is to find the destination or the information regarding the destination by controlled flooding of the RREQ across the network to forward the packets from source to destination. The TTL value determines the maximum number of hops that the RREQ can go through. To use the ERS, the source node sets the TTL values of the RREQ to an initial TTL_START value and initiates the route discovery. If no reply is received within the discovery period, the source then increases the RREQs broadcast id and then re-initialize the RREQ with TTL value increased by TTL_INCREMENT value. This process of increasing TTL value continues until the TTL_THRESHOLD value is reached, beyond which the RREQ is broadcasted across the entire network till it reaches RREQ_RETRIES.

The ERS method has the following restrictions. If the destination node is far from the source node, then the source node has to broadcast multiple RREQ messages. Consequently, intermediate nodes have to receive and process this message repeatedly. This leads to more consumption of energy and routing overhead.

\section{EXISTING WORK}

In existing system [8], RMECR and RMER algorithms describe the procedure that each node should undertake to find MECP, for which they require each node to have a complete image of the network topology. In ad hoc networks, this could be achieved using a link state proactive routing protocol such as optimized link state routing (OLSR). Using these algorithms we can compute energy cost of routes for hop-by-hop (HBH) and end-to-end (E2E) systems. RMECR is used to find the minimum cost routes. It considers remaining battery energy of node and reliability of links. RMECR finds energy efficient and reliable routes that increase the network lifetime. It provides hop-by-hop (HBH) and endto-end (E2E) reliability. Medium Access Control (MAC) layer is used to provide HBH reliability and CSMA and MACA is used to ensure the E2E reliability of the links. RMER minimize the consumed energy of the E2E packet traversal and does not consider about the remaining battery energy of nodes in a network. It is used as a 
benchmark to evaluate energy efficiency of the RMECR algorithm.

\section{A. Reliable Routing Algorithms for HBH System}

Reliable routing algorithm for $\mathrm{HBH}$ system, we first analyze the energy cost of a path for forwarding a data to its destination. Here considering the impact of limited number of retransmissions through each link, the size of packet or data and ACK packets. The energy cost of a path is analyzed in four steps: 1. analyzing the expected transmission count of data and ACK packets, 2. analyzing the expected energy cost of a link, 3 . analyzing the link and path reliability, 4. analyzing expected energy cost of a path.

After analyzing the energy cost of a path, we design a routing algorithm for finding minimum energy cost path (MECP) between every two nodes in the network. Here Dijkstra's algorithm is used to find MECP in HBH system. Dijkstra's shortest path routing algorithm is show only heuristic solution for finding MECP. RMER is an energy efficient routing algorithm, which minimizing the total amount energy consumed to forward a packet from source node to destination node. RMER does not consider the remaining battery energy of nodes.

B. Reliable Routing Algorithms for E2E System

Reliable routing for E2E system also, we first analyze the energy cost of a path for forwarding a packet from source to its destination. Here consider the impact of E2E ACKs and ACK lost. In E2E system, the energy cost of a path depends on the number of times that the packet and its E2E ACK are forwarded. To determine the energy cost formulating the E2E reliability of path for data packets and E2E ACKs, then calculate the expected energy cost of a path. After analyzing the energy cost of a path, we calculate the shortest path (i.e., MECP) between nodes in a networks using Dijkstra's algorithm.

RMECR routing algorithm is consider the remaining battery energy of the two nodes consumed to transfer a packet across a link. In E2E system the energy consumed by the transmitting node to forward a packet and also energy consumed by the receiving node. RMER routing algorithm is does not consider the remaining battery energy of nodes in the networks and minimize the energy consumption of E2E packet traversal.

\section{PROPOSED WORK}

ERMECR and ERMER algorithms are proposed new energy-aware routing algorithms for increasing energy-efficiency, reliability, and network lifetime. These algorithms could be designed using a link state proactive routing protocol such as new optimized link state routing (NOLSR). Both these algorithms are providing $\mathrm{HBH}$ and $\mathrm{E} 2 \mathrm{E}$ reliability of system.

\section{A. ERMECR Algorithm}

ERMECR algorithm can increase the lifetime of network based on energy-efficiency and reliability. It first analyzes the energy cost of a path in four ways expected transmission count of data and ACK packets, analyzing total energy consumption of a link, analyzing link and path reliability and expected energy cost of a path. After analyzing the energy cost, we calculate Copyright to IJARCCE shortest path using Bellman-Ford algorithm. The Bellman-Ford algorithm provides high computational complexity other than Dilkstra's algorithm.

ERMECR can calculate the link weight using Bellman-Ford algorithm for finding MECP (Minimum Energy Cost Path). It considers the remaining battery energy of nodes in the network. The battery cost of a link in ERMECR for HBH system, the link weight in this algorithm is obtained as

$$
\begin{gathered}
W(u, v)=e_{u, v}\left(L_{d}\right) \\
=\frac{a_{u, v}\left(L_{d}\right)}{C_{u}}+\frac{b_{u, v}\left(L_{d}\right)}{C_{v}} \\
=\frac{L_{d}}{r} E\left[n_{u, v}\left(L_{d}\right)\right]\left(\frac{A_{u}+\frac{P_{u, v}}{k_{u}}}{C_{u}}+\frac{B_{v}}{C_{v}}\right)+ \\
\frac{L_{h}}{r} E\left[m_{v, u}\left(L_{h}\right)\right]\left(\frac{A_{v}+\frac{P_{v}, u}{k_{v}}}{C_{v}}+\frac{B_{u}}{C_{u}}\right)
\end{gathered}
$$

The battery cost of a link in ERMECR for E2E system, and link weight is calculated in two factors.

1. Neglect the effect of the E2E ACK on the expected energy cost. The link weight is

$$
\begin{gathered}
W(u, v)=e_{u, v}\left(L_{d}\right) \\
=\frac{\varepsilon_{u, v}\left(L_{d}\right)}{C_{u}}+\frac{\omega_{u, v}\left(L_{d}\right)}{C_{v}} \\
=\frac{L_{d}}{r}\left(\frac{A_{u}+\frac{P_{u, v}}{k_{u}}}{C_{u}}+\frac{B_{v}}{C_{v}}\right)
\end{gathered}
$$

2. Consider the impact of E2E ACK on the energy cost. The link weight is

$$
\begin{array}{r}
W(u, v)=e_{u, v}\left(L_{d}\right)+e_{v, u}\left(L_{e}\right) \\
=\frac{L_{d}}{r}\left(\frac{A_{u}+\frac{P u, v}{k_{u}}}{C_{u}}+\frac{B_{v}}{C_{v}}\right)+\frac{L_{e}}{r}\left(\frac{A_{v}+\frac{P v, u}{k_{v}}}{C_{v}}+\frac{B_{u}}{C_{u}}\right)
\end{array}
$$

\section{B. ERMER Algorithm}

ERMER algorithm is used to find routes which minimize the total energy for packet traversal. It does not consider the remaining battery energy nodes. Bellman-Ford algorithm is used to finding shortest path between the nodes in ERMER algorithm. In ERMER the link weight for $\mathrm{HBH}$ system is

$$
\begin{aligned}
& W(u, v)=\frac{L_{d}}{r} E\left[n_{u, v}\left(L_{d}\right)\right]\left(A_{u}+\frac{P_{u, v}}{k_{u}}+B_{v}\right)+ \\
& \frac{L_{h}}{r} E\left[m_{v, u}\left(L_{h}\right)\right]\left(A_{v}+\frac{P_{v, u}}{k_{v}}+B_{u}\right)
\end{aligned}
$$

The link weight for E2E system is calculated in two ways.

1. Neglect the effect of the E2E ACK on the expected energy cost. The link weight is

$$
\mathrm{W}(\mathrm{u}, \mathrm{v})=\frac{\mathrm{L}_{\mathrm{d}}}{\mathrm{r}}\left(\mathrm{A}_{\mathrm{u}}+\frac{\mathrm{P}_{\mathrm{u}, \mathrm{v}}}{\mathrm{k}_{\mathrm{u}}}+\mathrm{B}_{\mathrm{v}}\right)
$$

Consider the impact of E2E ACK on the energy cost. The link weight is

$$
\begin{aligned}
& \qquad W(u, v)=\frac{L_{d}}{r}\left(A_{u}+\frac{P_{u, v}}{k_{u}}+B_{v}\right)+ \\
& \frac{L_{e}}{r}\left(A_{v}+\frac{P_{v, u}}{k_{v}}+B_{u}\right)
\end{aligned}
$$


V.

IMPLEMENTATION AND RESULT

To evaluate the performance of ERMECR and ERMER algorithms, consider a network in which nodes are uniformly distributed in a square area. Nodes are assumed to be static. In our simulations, they compute the probability of error-free reception of packets of size $\mathrm{x}$ bit over a link. The packet format in the simulation model is based on IEEE 802.11 standard. Each transmitted packet on the physical link consists of three parts: a preamble, a physical layer header, and the payload which includes user data and headers from higher layers.

For each transmitted packet by $u,\left(A_{u}+\frac{P_{u, v}}{k_{u}}\right) \frac{x}{r}$ is deducted from its battery energy, where $\mathrm{x}$ is the packet size. For each received packet by $v, B_{v_{r}^{-}}^{-}$is deducted from its battery energy, where $0 \leq \mathrm{x} 1 \leq \mathrm{x}$ is the size of detected part of the packet. Furthermore, nodes consume a small amount of energy when they are idle (i.e., they do not transmit or receive any data or control packet) and when they sense the medium. More specifically, they assume the energy consumption at the idle mode is $\mathrm{k}_{\mathrm{idle}} \frac{\mathrm{Bu}}{\mathrm{r}}$ Tidle, where $T_{\text {idle }}$ is the duration that a node is idle, and $k_{\text {idle }}$ is a constant. They also assume that the energy consumption during channel sensing is $k_{\text {sense }} \frac{B_{u}}{r} T_{\text {sense }}$, where $T_{\text {sense }}$ is the duration of sensing the channel and $\mathrm{k}_{\text {sense }}$ is a constant. The deployed routing protocol is NOLSR in which Hello messages are sent periodically every $\mathrm{T}_{\text {hello }}$ seconds and topology control messages are transmitted every $\mathrm{T}_{\mathrm{tc}}$ seconds. For each node $\mathrm{u}$, consider 10 levels of transmission power starting from $15 \mathrm{~mW}$ and increasing in steps of $15 \mathrm{~mW}$ up to the maximum transmission power $\operatorname{Pmax}=150 \mathrm{~mW}$. The table I shows various parameter used in simulations.

TABLE I

VALUES OF VARIOUS PARAMETER USED IN SIMULATIONS

\begin{tabular}{|l|l|}
\hline \multicolumn{1}{|c|}{ Parameter } & \multicolumn{1}{|c|}{ Value } \\
\hline $\begin{array}{l}\text { Initial battery energy of each node } \\
(\mathrm{B})\end{array}$ & $100[\mathrm{~J}]$ \\
\hline Network area & $350 * 350\left[\mathrm{~m}^{2}\right]$ \\
\hline Path-loss exponent $(\mathrm{y})$ & 3 \\
\hline Data rate $(\mathrm{r})$ & $100[\mathrm{Kbps}]$ \\
\hline $\begin{array}{l}\text { Power consumption of transmitter } \\
\text { circuit }\left(\mathrm{P}_{\mathrm{t}}\right)\end{array}$ & $100[\mathrm{~mW}]$ \\
\hline $\begin{array}{l}\text { Power consumption of receiver } \\
\text { circuit }\left(\mathrm{P}_{\mathrm{r}}\right)\end{array}$ & $100[\mathrm{~mW}]$ \\
\hline $\begin{array}{l}\text { Maximum transmission power } \\
\left(\mathrm{P}_{\text {max }}\right)\end{array}$ & $150[\mathrm{~mW}]$ \\
\hline $\begin{array}{l}\text { Minimum transmission power } \\
\left(\mathrm{P}_{\text {min }}\right)\end{array}$ & $15[\mathrm{~mW}]$ \\
\hline $\begin{array}{l}\text { Maximum\# of transmissions in } \\
\text { HBH system(Qu }\end{array}$ & 7 \\
\hline Transmission range $\left(\mathrm{d}_{\text {max }}\right)$ & $70[\mathrm{~m}]$ \\
\hline $\begin{array}{l}\text { Data packet size }\left(\mathrm{L}_{\mathrm{d}}\right) \\
\text { MAC ACK packet size }\left(\mathrm{L}_{\mathrm{h}}\right)\end{array}$ & $512[\mathrm{byte}]$ \\
\hline \begin{tabular}{l} 
E2E ACK packet size $\left(\mathrm{L}_{\mathrm{e}}\right)$ \\
\hline
\end{tabular} & $240[\mathrm{bit}]$ \\
\hline
\end{tabular}

\begin{tabular}{|l|l|}
\hline Hello packet size $\left(\mathrm{L}_{\text {hello }}\right)$ & $96[$ byte] \\
\hline Battery death threshold $\left(\mathrm{B}_{\mathrm{th}}\right)$ & 0 \\
\hline $\begin{array}{l}\text { Maximum collision probability } \\
\left(\mathrm{Pc}_{\max }\right)\end{array}$ & 0.3 \\
\hline channel sensing time $\left(\mathrm{T}_{\text {sense }}\right)$ & $50[\mu \mathrm{s}]$ \\
\hline $\mathrm{K}_{\text {idle }}$ & 0.2 \\
\hline $\mathrm{K}_{\text {sense }}$ & 0.4 \\
\hline $\mathrm{T}_{\text {hello }}$ & $10[\mathrm{~s}]$ \\
\hline $\mathrm{T}_{\mathrm{tc}}$ & $20[\mathrm{~s}]$ \\
\hline
\end{tabular}

The performance of these ERMECR and ERMER algorithms will be effective compared to the previous algorithms. The fig 2. shows that energy utilization of ERMECR and ERMER algorithms.

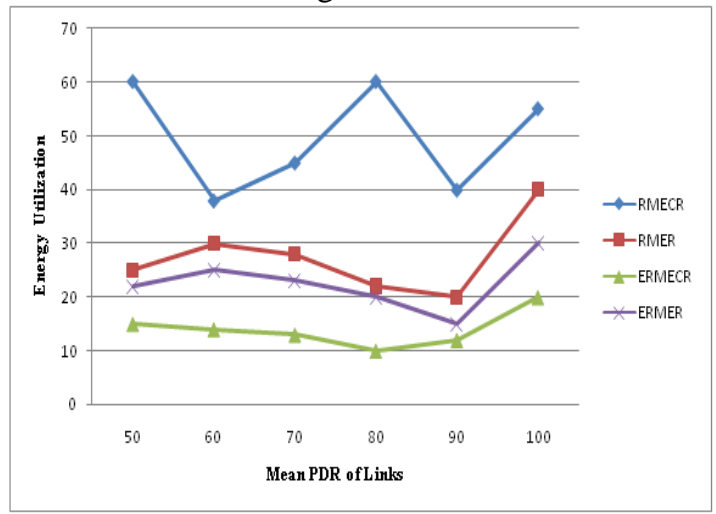

Fig 2. Energy Utilization

\section{CONCLUSION}

The proposed new energy aware routing algorithm, ERMECR can increase the lifetime of network using energy efficient and reliable route. In wireless ad hoc network ERMECR is used detailed energy consumption model for packet transfer. It considers the remaining battery energy of the nodes. ERMER algorithm is used find the minimum route cost for end-to-end packet traversal. Both these algorithms designed for hop-by-hop and end-to-end retransmission ensure the reliability. The performance of the proposed ERMECR and ERMER algorithms will be effective compared to the RMECR and RMER algorithms.

\section{REFERENCES}

[1] D.S.J. De Couto, D. Aguayo, J. Bicket, and R. Morris, "A HighThroughput Path Metric for Multi-Hop Wireless Routing," Proc. ACM MobiCom, pp. 134-146, 2003.

[2] S. Singh and C. Raghavendra, "PAMAS - Power Aware MultiAccess Protocol with Signalling for Ad Hoc Networks," ACM Computer Comm. Rev., vol. 28, pp. 5-26, 1999.

[3] J. Gomez-Castellanos, A. Campbell, M. Naghshineh, and C. Bisdikian, "PARO: A power-aware routing optimization scheme for mobile adhoc networks," draft-gomez-paro-manet-00.txt, work in progress. IETF, Mar. 2001

[4] C.k. Toh, H. cobband Scott, "Performance Evaluation of battery life aware routing schemes for wireless ad hoc networks", Proceedings of IEEE ICC'01, June 2001.

[5] S. Banerjee and A. Misra, "Minimum Energy Paths for Reliable Communication in Multi-Hop Wireless Networks," Proc. ACM MobiHoc, pp. 146-156, June 2002 
[6] Q. Dong, S. Banerjee, M. Adler, and A. Misra, "Minimum Energy Reliable Paths Using Unreliable Wireless Links," Proc. ACM MobiHoc, pp. 449-459, May 2005.

[7] X.-Y. Li, Y. Wang, H. Chen, X. Chu, Y. Wu, and Y. Qi, "Reliable and Energy-Efficient Routing for Static Wireless Ad Hoc Networks with Unreliable Links," IEEE Trans. Parallel and Distributed Systems, vol. 20, no. 10, pp. 1408-1421, Oct. 2009.

[8] J. Vazifehdan, R. Prasad, and I. Niemegeers, "Energy-Efficient Reliable Routing Considering Energy in Wireless Ad Hoc Networks," IEEE transactions on mobile computing, vol. 13, No.2, Feb. 2014.

[9] D.N Pham, and H. Choo, "Energy Efficient Ring Search for Route Discovery in MANETs," IEEE International Conference of Communication, Turkey, 2008.

\section{BIOGRAPHIES}

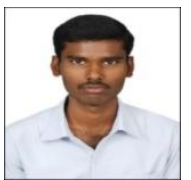

Mr. S. Agilan obtained his bachelor's degree in Information Technology from Adhiyamaan college of Engineering affiliated to Anna University, Chennai. Currently he is pursuing his Master degree in Computer Science and Engineering at Adhiyamaan College of Engineering, Hosur, Tamilnadu.

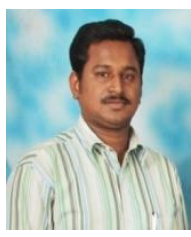

Prof. B. Gopinathan obtained his bachelor's degree in Information Technology from M.I.E.T Engineering College, Trichy-7 and he received his Master degree in Computer Science and Engineering from Arunai Engineering College, Tiruvannamalai. Currently he is pursuing his $\mathrm{Ph} . \mathrm{D}$ at Anna University, Chennai, in the field of Ad hoc networks. He has 8 years of teaching experience and currently, he is working as a Associate Professor in CSE Department at Adhiyamaan college of Engineering, Hosur, Tamilnadu. 